\title{
Reduction of Arterial Oxygen Saturation Among Rescuers During Cardiopulmonary Resuscitation in a Hypobaric Hypoxic Environment
}

\author{
Takashi Suto, MD, PhD; Shigeru Saito, MD, PhD; Masaru Tobe, MD, PhD; \\ Masafumi Kanamoto, MD, PhD; Yusuke Matsui, MD \\ Department of Anesthesiology, Gunma University Graduate School of Medicine, Maebashi, Japan
}

\begin{abstract}
We experienced a case involving prolonged cardiopulmonary resuscitation (CPR) during cardiac arrest on Mt. Fuji (3776 m), demanding lengthy exertion by the rescuers performing CPR. Considering the effects of exertion on the rescuers, we examined their percutaneous arterial oxygen saturation during simulated CPR and compared the effects of compression-only and conventional CPR at $3700 \mathrm{~m}$ above sea level. The effects of CPR on the physical condition of rescuers were examined at the summit of Mt. Fuji: three rescue staff equipped with pulse-oximeters performed CPR with or without breaths using a CPR mannequin. At $3700 \mathrm{~m}$, the rescuers' heart rate increased during CPR regardless of the presence or absence of rescue breathing. Percutaneous arterial oxygen saturation measured in such an environment was reduced only when CPR without rescue breathing was performed. Scores on the Borg scale, a subjective score of fatigue, after CPR in a $3700 \mathrm{~m}$ environment were 13 to 15 of 20 (somewhat hard to hard). Performing CPR at high altitude exerts a significant physical effect upon the condition of rescuers. Compression-only CPR at high altitude may cause a deterioration in rescuer oxygenation, whereas CPR with rescue breathing might ameliorate such deterioration.
\end{abstract}

Keywords: high altitude, alpine accidents, mountain rescue, basic life support

\section{Introduction}

A growing number of individuals with cardiovascular disease are ascending to high altitude, ${ }^{1}$ thus increasing the potential for cardiac arrest in the field. Immediate cardiopulmonary resuscitation (CPR) is required in patients with cardiac arrest. ${ }^{2,3}$ However, the performance of CPR is effort-intensive even at sea level, and CPR at altitude is even more strenous. ${ }^{4-6}$

We previously described the application of prolonged CPR in a wilderness setting at high altitude on a trekker who developed cardiac arrest. The rescuer, who continued CPR during steep mountain transportation even though CPR in the lopsided, narrow carriage was very difficult, became exhausted after the work. ${ }^{6}$ Following the

Corresponding author: Shigeru Saito, MD, PhD, Department of Anesthesiology, Gunma University Graduate School of Medicine, 3-3915, Showa-machi, Maebashi, 371-8511, Japan.;

e-mail: shigerus@ gunma-u.ac.jp.

Submitted for publication May 2019.

Accepted for publication October 2019. aforementioned actions, we investigated the physical status of rescuers who provide CPR at high altitudes and found a significant impact on physiological parameters. ${ }^{6}$ The Wilderness Medical Society practice guidelines for the out-ofhospital evaluation and treatment of accidental hypothermia advise that CPR can be stopped if it is futile. Additionally, CPR can be interrupted to facilitate evacuation and can be stopped due to rescuer exhaustion. ${ }^{7}$

The most recent American Heart Association (AHA) guidelines state that compression-only CPR is simpler for lay providers to learn than conventional CPR (compressions with rescue breathing) and can even be coached by a dispatcher during an emergency. The AHA also determined that public education on compression-only CPR promoted the use of both overall CPR and compression-only CPR by bystanders. ${ }^{8}$ Conversely, the opinion has been put forward that compression-only CPR is more exhausting than conventional CPR. It is reported that chest compressions to an appropriate depth decrease more rapidly during chest compression-only CPR owing to rescuer fatigue. ${ }^{9} \mathrm{We}$ also suspected that compression-only CPR and 
conventional CPR would have different physiological effects on rescuers, especially at high altitudes. Taking deep breaths prior to exhaling into a victim and the action of blowing against expiratory resistance (resistance of the airways and lungs of victims) seem to significantly affect the respiratory and circulatory status of would-be rescuers.

\section{Methods}

Data were obtained from trainees who voluntarily practice CPR under hypobaric conditions, in the expectation of a need for basic life support action when planning high altitude treks. Approval of the local human ethics committee (Gunma University research ethics committee for human studies no. 20-20) was obtained prior to analysis, and the protocol was registered in an internationally accessible clinical research database (UMIN; UMIN000020659) as an observational case study.

At the summit hut of Mt. Fuji, located at 3700 m, 3 male volunteers participated in this study. Participants had completed a basic life support and CPR training course (adapted according to international liaison committee on resuscitation guidelines $)^{3}$ before the field activity. All participants were office workers with no regular physical exercise regimen, and none had been exposed to altitudes over $2000 \mathrm{~m}$ during the half-year before the CPR practice. They passed prestudy health checkups, including chest x-ray, electrocardiography, and laboratory blood tests, and none had medical ailments such as cardiovascular or pulmonary disease.

Barometric pressure at altitude was $630 \mathrm{hPa}$, according to the local meteorological center at the time of field work. For measurements at $3700 \mathrm{~m}$, participants were transported to an altitude of $2400 \mathrm{~m}$ by car and then ascended the last $1300 \mathrm{~m}$ on foot, while carrying a load of approximately $5 \mathrm{~kg}$. Transportation by car from sea level to an altitude of $2400 \mathrm{~m}$ took approximately $4 \mathrm{~h}$. The total duration required for the ascent from sea level to $3700 \mathrm{~m}$ was approximately 6 to $8 \mathrm{~h}$. All measurements were obtained in wind-sealed constructions, and the temperature was maintained at $18^{\circ} \mathrm{C}$. After arrival at the new altitude, participants were permitted 30 min of rest before performing CPR.

These 3 volunteers, who were training to be efficient high altitude rescuers, had arterial oxygen saturation and heart rate (HR) measured during practice of $\mathrm{CPR}$ at 3700 $\mathrm{m}$. In the field work, the volunteers performed CPR after climbing, as expected in a real accident. They practiced both compression-only CPR and CPR with breath. At the summit of Mt. Fuji, we used a handheld oximeter designed for portability and durability (TuffSat; GE Healthcare, Helsinki, Finland). A probe for measuring percutaneous arterial oxygen saturation $\left(\mathrm{SpO}_{2}\right)$ was always positioned on a finger because an earlier pilot test had shown that measurement during CPR was more stable with the probe on the index or middle finger than on an ear or toe. HR and arterial blood oxygen saturation were recorded every $15 \mathrm{~s}$.

Borg scale scores were collected immediately after each CPR activity. ${ }^{10}$ This simple scale of perceived exertion has often been used to regulate exercise intensity. A higher Borg scale score indicates a subjective feeling of greater physical intensity.

CPR was performed continuously as a single-operator procedure using a transportable CPR trainer (MiniAnne; Laerdal Japan, Tokyo, Japan) and according to the 2015 AHA Guidelines. ${ }^{8}$ The mannequin was not wearing outdoor clothing, but the rescuers were clothed. The simulator was placed on a flat, wooden floor in the mountain hut. Compression-only and conventional CPR procedures were sequentially repeated twice without break, and the duration of each mode was $2 \mathrm{~min}$. The rescuers then performed CPR for 8 min without resting. The rate of chest compressions was kept constant at 100 compressions $\cdot \min ^{-1}$ using a digital quartz metronome (CN89; Yamaha Musical Trading, Tokyo, Japan). The adequacy of chest compressions was monitored by measuring chest balloon pressures using a pressure gauge (CE0123; VBM, Sulz, Germany) connected to the balloon inflation valve. Study participants were advised to attain a peak chest balloon pressure of 30 to $50 \mathrm{~cm} \mathrm{H}_{2} \mathrm{O}$ with each compression. This pressure value was determined by observing that a 4 - to $5-\mathrm{cm}$ chest compression on the simulator model produced results within this pressure range on the gauge (the depth of chest compression recommended in the 2015 AHA Guidelines is $5-6 \mathrm{~cm}){ }^{8}$ During CPR with breaths, the adequacy of breathing was monitored by observing full inflation of the simulated lung. A compression:ventilation ratio of 30:2 was used, in accordance with the 2015 AHA guidelines. ${ }^{8}$

The obtained data were given to the high altitude data analysis group working on "Health survey of the hypobaric hypoxic environment visitors," an academic research project collecting biometric data from alpine tourists, to propose adequate health care during alpine hikes. Data are presented as mean $\pm \mathrm{SD}$ with range, as appropriate.

\section{Results}

Participants were $43 \pm 8(36-54)$ y in age, $168 \pm 2 \mathrm{~cm}$ in height, and $59 \pm 1 \mathrm{~kg}$ in mass. They performed CPR for 8 minutes continuously. $\mathrm{HR}$ and $\mathrm{SpO}_{2}$ measured before and during CPR by the 3 participants are shown in Figure 1. HR immediately before the CPR was 95, 85, 123 beats $\cdot \min ^{-1}$ in Rescuers 1,2 , and 3, respectively. The maximal HR value was 128,142 , and 193 beats $\cdot \min ^{-1}$ in Rescuers 1, 2, and 3, respectively. HR increased during CPR in all 3 participants. The difference between maximal and minimal HR was 34,57 , and 94 beats $\cdot \min ^{-1}$ in 


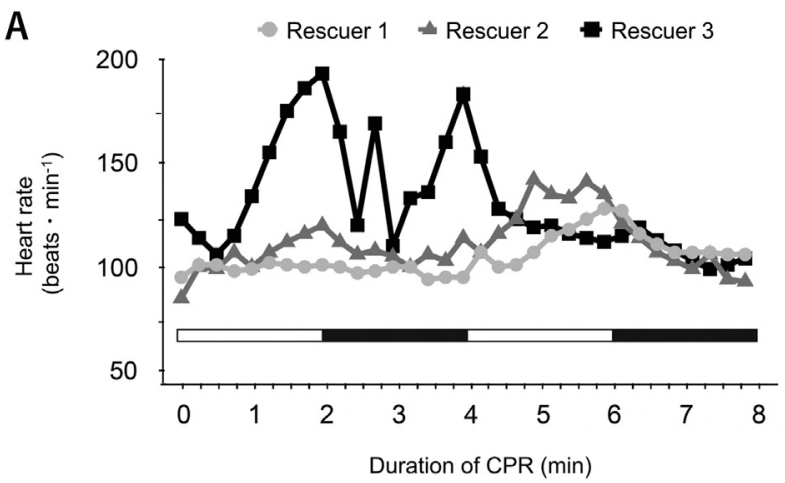

B



Figure 1. Changes in heart rate (A) and percutaneous arterial oxygen saturation (B) in 3 participants performing cardiopulmonary resuscitation at an altitude of $3700 \mathrm{~m}$, on the summit of Mt. Fuji. White and black bars indicate periods for which cardiopulmonary resuscitation was performed in compression-only and with-breaths modes, respectively.

Rescuers 1, 2, and 3, respectively. The HR in Rescuer 3 was extremely elevated during the early half of CPR practice; the maximum HR recommended for the age $(220-36)$ was exceeded in Rescuer 3. However, the mode of CPR (compression-only or with breaths) did not affect changes in HR. The value in Rescuer 1 and Rescuer 2 increased during the second, compression-only CPR.

$\mathrm{SpO}_{2}$ immediately before the CPR was 89,86 , and $87 \%$ in Rescuers 1, 2, and 3, respectively. The lowest value was 81, 80, and 77\% in Rescuers 1, 2, and 3, respectively. $\mathrm{SpO}_{2}$ was reduced during compression-only CPR in all participants, and the trend was most obvious in Rescuer 3. The difference between maximal $\mathrm{SpO}_{2}$ and minimal $\mathrm{SpO}_{2}$ was 8,11 , and $12 \%$ in Rescuers 1, 2, and 3, respectively.

The Borg scale score was 13 in Rescuer 1 and Rescuer 2, regardless of CPR mode. In Rescuer 3, scores during compression-only CPR and CPR with breaths were 15 (meaning hard or heavy) and 13 (meaning somewhat hard), respectively.

Because compression pressure was continuously monitored and the rhythm was indicated by metronome, the quality of the CPR in terms of adequacy of compression depth and frequency was confirmed by the rescuers and the attending observer. The 3 rescuers followed advanced cardiovascular life support (ACLS) guidelines, and quality did not decrease with time. The rescuers could meet the 30:2 compression ratio and ensured the chest rose and fell adequately with breaths. Rescuer 3's CPR quality did not decrease during profound desaturations into the mid-70s.

\section{Discussion}

The present findings indicate that CPR in a hypoxic environment significantly affects the physiological parameters of providers. In particular, compression-only CPR seems likely to decrease oxygenation among rescuers.

We previously showed that applying CPR places major demands on the body at high altitude. ${ }^{6}$ The degree of reduction in $\mathrm{SpO}_{2}$ observed in the present study was comparable to the values measured at an altitude of $3700 \mathrm{~m}$ in our previous study. ${ }^{6}$ The relatively wide deviation in $\mathrm{SpO}_{2}$ among CPR providers was also comparable. Values for $\mathrm{SpO}_{2}$ vary among hikers at any given altitude. ${ }^{1,5,6}$ The frequency and mean of breathing both affect $\mathrm{SpO}_{2}$, more so at high altitude than at sea level, according to the degree alveolar $\mathrm{CO}_{2}$ partial pressure and positive end-expiratory pressure. When the breathing frequency is relatively low, $\mathrm{CO}_{2}$ partial pressure increases and alveolar $\mathrm{O}_{2}$ partial pressure decreases, which induces lower $\mathrm{SpO}_{2}$. When the positive end-expiratory pressure effect is intentionally or unintentionally provoked by each participant via pursed-lips expiration, $\mathrm{O}_{2}$ passes through the alveolar membrane more effectively, is loaded on hemoglobin, and induces higher $\mathrm{SpO}_{2}$ levels. These phenomena are not apparent at sea level, where ambient $\mathrm{O}_{2}$ pressure is not low. Furthermore, when $\mathrm{O}_{2}$ consumption and $\mathrm{CO}_{2}$ production increase during exercise, the reduction in $\mathrm{SpO}_{2}$ becomes more obvious. The information derived from the present study in the field is consistent with our hypothesis that CPR with breath might improve oxygenation in rescuers when compared with compression-only CPR at high altitude. The increase in HR among rescuers must be derived from an increase in oxygen demand from CPR.

Applying CPR has both physiological and mental effects, including feelings of fatigue. Scores on the Borg scale were high at $3700 \mathrm{~m}$, where $\mathrm{SpO}_{2}$ decreased and HR increased. This is consistent with previous findings. ${ }^{4}$ The latest guidelines recommend switching the personnel applying chest compressions every $2 \mathrm{~min}$, based on findings of fatigue and an accompanying loss of CPR quality. ${ }^{8}$ In particular, rescuers should switch every minute to maintain the quality of chest compression during compressiononly CPR. ${ }^{9}$ In remote, high altitude, out-of-hospital environments, few passers-by or colleagues might be able to apply CPR. Therefore, CPR is unlikely to be applied according to recommendations in such environments. 


\section{LIMITATIONS}

This study had limitations. The small number of participants limited generalizability.

The mannequin simulator might not represent a person in cardiac arrest at altitude. The sheltered environment might not reflect actual evacuation circumstances. The participants rested before CPR, which may not reflect real-life circumstances. This report is intended to be hypothesisgenerating, and the authors are not aiming to change ACLS protocols. Further research is recommended. Although our experimental conditions differed from a real rescue scene, a recent publication does describe the development of apparent hypoxia among CPR practitioners applying compression-only CPR in a hypobaric simulation chamber. $^{11}$

\section{Conclusions}

We compared the effects of CPR with or without breaths on cardiorespiratory dynamics at $3700 \mathrm{~m}$. The work of CPR had a significant impact on the physiological parameters of participants. Compression-only CPR at high altitude may deteriorate rescuer oxygenation, whereas CPR with breaths might ameliorate such deterioration. Because CPR demands high exertion, especially at high altitude, CPR with breaths may be the preferred method. Because this is a case report with simulation, further research is required before CPR recommendations can be made.

Author Contributions: Data acquisition (TS, SS, MT, MK, YM); data analysis (TS, SS, MT); manuscript writing (TS, SS); critical review (TS, SS, MT, MK, YM)

Financial/Material Support: This work was funded by the Japanese Ministry of Education and Science with JSPS grants to Shigeru Saito (Nos. 16H02678, 16K15673).

Disclosures: None.

\section{References}

1. Keyes LE, Mather L, Duke C, Regmi N, Phelan B, Pant S, et al. Older age, chronic medical conditions and polyphar- macy in Himalayan trekkers in Nepal: an epidemiologic survey and case series. J Travel Med. 2016;23(6):1-6.

2. Berg RA, Hemphill R, Abella BS, Aufderheide TP, Cave DM, Hazinski MF, et al. Part 5: adult basic life support: 2010 American Heart Association guidelines for cardiopulmonary resuscitation and emergency cardiovascular care. Circulation. 2010;122(18 Suppl 3):S685-705.

3. Kleinman ME, Brennan EE, Goldberger ZD, Swor RA, Terry M, Bobrow BJ, et al. Part 5: adult basic life support and cardiopulmonary resuscitation quality: 2015 American Heart Association guidelines update for cardiopulmonary resuscitation and emergency cardiovascular care. Circulation. 2015;132(18 Suppl 2):S414-35.

4. Wang JC, Tsai SH, Chen YL, Hsu CW, Lai KC, Liao WI, et al. The physiological effects and quality of chest compressions during CPR at sea level and high altitude. Am J Emerg Med. 2014;32(10):1183-8.

5. Suto T, Saito S. Considerations for resuscitation at high altitude in elderly and untrained populations and rescuers. Am J Emerg Med. 2014;32(3):270-6.

6. Narahara H, Kimura M, Suto T, Saito H, Tobe M, Aso C, et al. Effects of cardiopulmonary resuscitation at high altitudes on the physical condition of untrained and unacclimatized rescuers. Wilderness Environ Med. 2012;23(2):161-4.

7. Zafren K, Giesbrecht GG, Danzl DF, Brugger H, Sagalyn EB, Walpoth B, et al. Wilderness Medical Society practice guidelines for the out-of-hospital evaluation and treatment of accidental hypothermia. Wilderness Environ Med. 2014;25(4): 425-45.

8. American Heart Association. 2015 American Heart Association guidelines update for CPR and ECC, 2015. Available at: https://www.cercp.org/images/stories/recursos/Guias\% 202015/Guidelines-RCP-AHA-2015. Accessed June 26, 2019.

9. Nishiyama C, Iwami T, Kawamura T, Ando M, Yonemoto N, Hiraide A, et al. Quality of chest compressions during continuous CPR; comparison between chest compression-only CPR and conventional CPR. Resuscitation. 2010;81(9):1152-5.

10. Borg GA. Psychophysical bases of perceived exertion. Med Sci Sports Exerc. 1982;14(5):377-81.

11. Sato T, Takazawa T, Inoue M, Tada Y, Suto T, Tobe M, et al. Cardiorespiratory dynamics of rescuers during cardiopulmonary resuscitation in a hypoxic environment. Am J Emerg Med. 2018;36(9):1561-4. 